\title{
Radio Computer Communications Network for DisABLED People
}

\author{
András Arató, Teréz Vaspöri \\ KFKI Research Institute for Measurement \\ and Computing Techniques
}

\begin{abstract}
Communication is essential for people with all kinds of disabilities. Blind people can access more information, deaf people can have conversation with others, mobility impaired people can also access information easier. What to do in a country like Hungary which has a shortage of telephone lines? This article describes a possible solution through extending two-way radio to the internet.
\end{abstract}

\section{The Importance of Computer Communications:}

Prof. Norman Coombs a blind history teacher at the Rochester Institute of Technology NY USA made a presentation about telecommunication and education of disabled students at the 2nd ICCHP in Zurich [1]. This meeting played an important role in modifying our activity in the development of assistive devices. Our BraiLab talking computers [2] can be used more effectively by blind users if they have communication facilities. During a short visit of prof. Coombs in Hungary in 1993 we demonstrated the usefulness of computer communication not only for blind people but also for the community of deaf and mobility impaired people.

Using the Brailab PC Hungarian visually impaired users can have access to information from the Teletext broadcast of Television. Several data bases are also available on CD-ROM (e.g. Hungarian Laws, Telephone Book) with special application environment of the intelligent BraiLab screen reader program. Many data bases are accessible only via internet. A late development of talking telex could have helped blind people but the use of telex is decreasing in Hungary too.

Deaf people in Hungary use only text telephones as electronic communication devices. The level of education of hearing impaired persons is rather low unfortunately. This is not due to their intelligence level but due to our limited possibilities. We hope that if we will use computer communication for education it will help greatly.

Mobility impaired people have already been using computers for their rehabilitation. There were organized working places for data entry. Only floppy disks are used for "data transfer".

A normal way for connecting users to network resources would be telephone modems. Unfortunately there is a shortage of telephone lines in Hungary so you 
often have no phone line for voice transmission to say nothing about data transfer. To overcome this situation we decided to build a radio communications network.

\section{The Center for Disabled Students Services}

Prof. Laszlo Varga initiated the education of visually and mobility impaired students at the Budapest Eotvos Lorand University from the 80s till the present. He is the head of the Computer Science department of the Faculty of Natural Sciences.

Digital Equipment Corporation helped to build a computer center for blind students with its European Contributions Program. A VAX computer is the main resource while 7 PC-s equipped with DECtalk speech synthesizers serve as terminals. All computers are connected to the Ethernet of the University. From the University Local Area Network there is an access to the internet too. This University center will serve as the nucleus of radio communications network for disabled students.

Blind users have already learned how to access the internet with Telnet, FTP (File Transfer Protocol), Finger, Ping etc. They enjoy it very much. There is a Braillo braille printer with Hungarian braille publishing system and the Recognita OCR for their services [3]. As all the students have their own PC-s with Brailab PC talking adapters they would like to have access to network facilities from home.

\section{The Packet Radio Principle}

The authors of this article are radio amateurs (our callsigns are HG5BDU and HG5BDR) so we are familiar with AX25 packet radio techniques. Packet radio was first created by Vancouver amateurs in Canada. They used fixed addressing of possible 256 different stations. American amateurs built the AX25 standard with extended addressing and similar features to X25 packet switching standards. Callsigns are used for station addresses. There is also the possibility to use several callsigns for designating packet repeaters showing the route in the header.

Later special node software was developed (NET/ROM, TheNet Node) for the 3rd level of ISO OSI model. Protocols are implemented in 8 bit computer codes mostly for Z80 microprocessor so devices are very cheap. A typical packet radio terminal consists of a PC, Terminal Node Controller (TNC), radio transceiver and antenna. The TNC is an intelligent modem containing the AX25 protocol implementation and the modem hardware. TNC does the packet assembling disassembling so it is actually a PAD for AX25.

The most commonly used modem is 1200 baud Audio Frequency Shift Keying (AFSK) which is a standard used in telephony. For higher speeds Frequency Shift Keying (FSK) is used. AFSK modems can be plugged in the speaker and microphone connectors of the radio while FSK requires special plugs for modulator and demodulator of VHF or UHF FM transceivers.

A milestone occurred in packet radio development when Phil Karn developed his first TCP/IP software for personal computers and AX25 in 1989. His callsign 
is well known not only among radio amateurs but at universities too. The Technical University of Vienna also used KA9Q tcp/ip package for their routing purposes. This software is free for educational and amateur use. There are several variants of this software. Descriptions will be found further in this article.

Edwin Brownrigg and Dewayne Hendricks made a packet radio demo in Budapest in the buildings of the Hungarian Academy of Sciences in October 6, 1992. The goal of the Budapest project was to demonstrate the technical feasibility of using packet radio as an interface to the Internet, thus establishing the potential for low cost international connectivity for the Central European Agricultural Libraries. Unfortunately the authors of this paper did not know about this demo at that time.

\section{The First Experimental Radio Network in KFKI}

A PC gateway has been set up in KFKI research center since March 1990. This firs gateway could be crossed only from radio side to Ethernet. A radio amateur software SP (made by S. Kluger DL1MEN) was modified to accept special command implementing CTERM protocol for DECnet. CTERM is a remote terminal protocol in Digital's network. The //KFKI command contained the DECnet hostname, accountname and password for connecting to the proper host.

The schematic diagram of the KFKI radio Ethernet gateway experiment looks like:

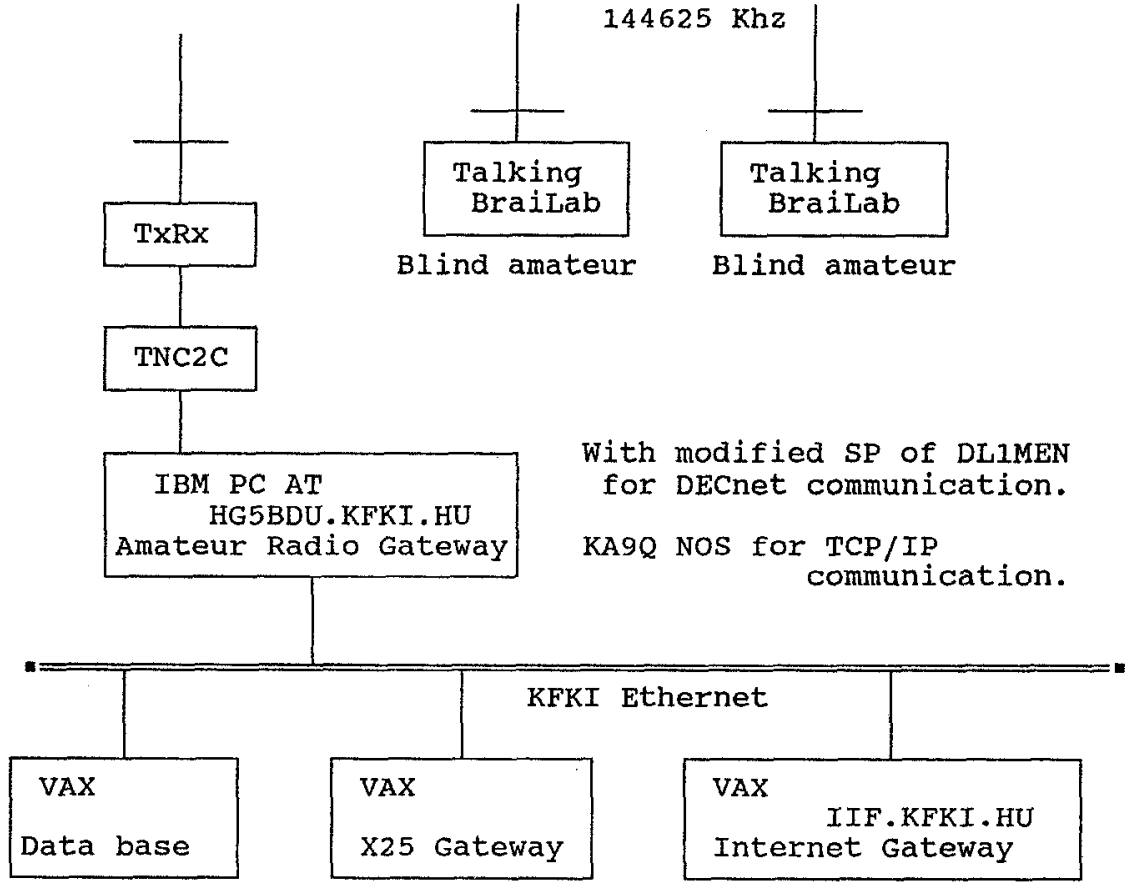


After internet connections were established the SP software was changed to KA9Q. At present the so called JNOS version of gateway software is running. The developer of this version is Johan K. Reinalda (WG7J/PA3DIS). The features of this Gateway and Bulletin Board System (BBS) are very rich. You can go not only from the radio side to the internet and vice versa but it is a full service amateur BBS with internet Simple Mail Transfer Protocol (SMTP), Network News Transfer Protocol (NNTP), FTP, Post Office Protocol (POP) etc.

JNOS can serve as a router from and to the radio side and the mail gateway too. JNOS is developed from KA9Q NOS (Network Operating System) [4]. NOS means that all client and service processes are handled by an operating system under MS-DOS. From radio side you can use simple AX25 protocol or TCP/IP protocol. TCP/IP can be routed through existing AX25 VHF/UHF radio networks. With AXIP protocol, the internet is transparent for the AX25 and for TheNet protocol. When $\mathrm{KA} 9 \mathrm{Q}$ is working in a $\mathrm{PC}$, there is a very simple protocol is loaded in the TNC (KISS Keep It Simple Stupid). AX25 protocol implementation is written in $\mathrm{C}$ in NOS.

The amateur society has the large A class 44.x.x.x internet address. This is a very special network which is distributed all over the world. Therefor a special routing called encapsulating is developed for routing 44.X.X.X addresses. The whole internet is viewed as transparent network for the 44.x.x.x addresses.

There was a unique feature developed for NOS: the Converse. All gateway systems form a large on line conference network. They use the converse protocol for exchanging lines all over the world. If you can not meet your partner on line you can of course write letters, bulletins or network news.

\section{The Plan of the Full Duplex Radio Network}

As a next step we would like to build a PR radio network using a simplex 9600 baud radio channel. The KFKI research center is on the top of a 500 meter high hill in Csilleberc. It can be reached from the hill. As the number of users grows, we should change the simplex channel to full-duplex one. We will use G3RUH's 9600 baud FSK modem for full-duplex operation.

For this solution two $25 \mathrm{KHZ}$ step channel is required for the radio communication. We would gladly widen this network for other countries, where handicapped persons need computer communications as an equalizer factor in their learning. We have experimented with NOS in radio amateur band. The Hungarian Ministry of Transport and Communications promised a free channel for blind people in the professional band. We do hope that later we can realize the usefulness of our proved technique for the wider handicapped user community.

Why full-duplex operation is so important? The answer is in the "hidden transmitter" phenomenon. On simplex channel station A sends a packet for B, addressed to $\mathrm{C}, \mathrm{B}$ decodes the packet and hands it on to $\mathrm{C}$ on the same radio channel. The disadvantages are that each retransmission after collision by the repeater station B blocks the single radio channel. Also, A and C and perhaps other stations on the channel cannot hear each other's transmissions. As a result, 
their packet may collide and get lost. All this affects the throughput. Networks based on single radio channel digipeaters are a waste of time, especially when one expects heavy traffic.

In regenerating repeaters the data signal is decoded into a bit stream, which get immediately re-encoded and retransmitted on another frequency. Good regenerating repeaters also regenerate the TX clock, i.e., the rate at which the bits come in. This permits a near-perfect output signal stripped of almost all noise. If the packet was decodable at the repeater site, it will almost certainly be decodable at its destination as well [6].

User stations work in half-duplex mode with shifted frequencies for transmitting and receiving. All stations have to be compatible only with the central repeater station and can use directional antennas still they can detect a carrier from every station for proper CSMA mode of operation (Carrier Sensing Multiple Access).

Terminals of the system in KFKI will use $434.200 \mathrm{MHZ}$ for receiving and $432.600 \mathrm{MHZ}$ for transmitting. This 1.6 MHZ shift is rather small so special filters and separate antennas have to be used at the full duplex repeater.

The system schematics diagram with full duplex repeater will look like:

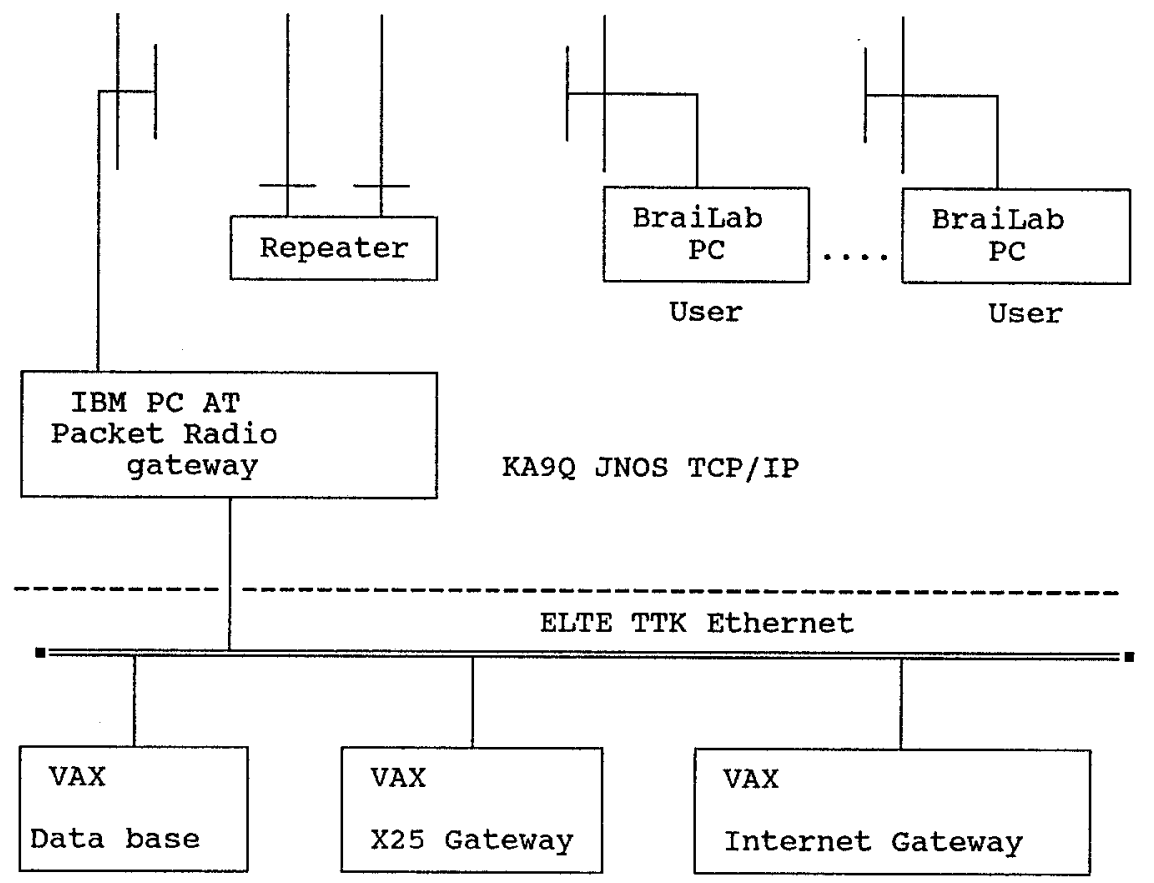

Users of this system will be able to reach packet radio gateway as BBS with Telnet function or as a mail gateway with SMTP. They will be able to use internet sources from their transportable radio terminals equipped with small directional antennas even from home or from anywhere in the town. This will be a small step towards personal communication. Ten packet radio tcp/ip terminals will be 
given to blind, deaf and mobility impaired radio amateurs to test and learn their new possibilities.

There is an Information Program for Handicapped radio- Amateurs to help in satisfying special needs for people to pass exam and work as HAMs. I.P.H.A. is coordinated by $\mathrm{A}$. Tobbe (PA3ADR) in the Netherlands.

\section{Conclusions}

People with disabilities have more need for the digital communications for their studying and integration into the society than able persons. The computer communications and assistive devices equalize them with the able bodied. When you can not use land line cables you can try to use packet radio networks.

Network communication can help in distributing free software, computer documentation, tutorials for the handicapped persons. A blind computer programmer can discuss problems with a deaf colleague in different countries using remote computer conferencing. The amateur radio development in this field could change the life of many handicapped people in Central and Eastern Europe.

\section{Acknowledgements}

The radio communication project was funded be IIF 'program. Assistive devices research was funded by OTKA. Thanks for Norman Coombs for helping to write this article.

\section{Literature}

[1] Norman Coombs, Telecommunications, Education and the Handicapped. 2nd ICCHP Zurich 1990. p 51-54.

[2] Andras Arato \& Terez Vaspori, Synthetic Speech for the Blind Integrated with Intelligent Screen Reader. 3rd ICCHP Vienna 1992. p 8-13.

[3] A. Arato, P. Molnar, T. Vaspori, Computer Aided Hungarian Contracted Braille. 2nd ICCHP Zurich 1990. p 1-6.

[4] Ian Wade, NOSintro TCP/IP over Packet Radio An Introduction to the KA9Q Network Operating System. Dowermain Ltd. Bedfordshire United Kingdom 1992. p 356.

[5] A. Tobbe Information Programme for Handicapped radio- Amateurs. I.P.H.A. 1990 I.A.R.U. Region 1.

[6] Ulrich Guenther ZLLDDL, 10 Feb 94 06:29:25 GMT From: news-mailgateway@ucsd.edu 